\title{
Percutaneous pericardiocentesis for pericardial effusion: predictors of mortality and outcomes
}

\author{
Andrea Pennacchioni ${ }^{1}$ - Giulia Nanni ${ }^{1}$ - Fabio Alfredo Sgura ${ }^{1}$. Jacopo Francesco Imberti ${ }^{1}$. \\ Daniel Enrique Monopoli ${ }^{1}$. Rosario Rossi ${ }^{1}$ - Giuseppe Longo ${ }^{2}$. Salvatore Arrotti ${ }^{1}$ - Marco Vitolo ${ }^{1,3}$. \\ Giuseppe Boriani ${ }^{1}$ (1)
}

Received: 10 November 2020 / Accepted: 13 January 2021 / Published online: 22 February 2021

(c) Società Italiana di Medicina Interna (SIMI) 2021

\begin{abstract}
Pericardial effusion can dangerously precipitate patient's hemodynamic stability and requires prompt intervention in case of tamponade. We investigated potential predictors of in-hospital mortality, a composite outcome of in-hospital mortality, pericardiocentesis-related complications, and the need for emergency cardiac surgery and all-cause mortality in patients undergoing percutaneous pericardiocentesis. This is an observational, retrospective, single-center study on patients undergoing percutaneous pericardiocentesis (2010-2019). We enrolled 81 consecutive patients. Median age was 71.4 years (interquartile range [IQR] 58.1-78.1 years) and 51 (63\%) were male. Most of the pericardiocentesis were performed in an urgency setting (76.5\%) for cardiac tamponade (77.8\%). The most common etiology was idiopathic (33.3\%) followed by neoplastic (22.2\%). In-hospital mortality was $14.8 \%$ while mortality during follow-up (mean 17.1 months) was $44.4 \%$. Only hemodynamic instability (i.e., cardiogenic shock, hypotension refractory to fluid challenge therapy and inotropes) was associated with in-hospital mortality at the univariate analysis (odds ratio [OR] 7.2; 95\% confidence interval [CI] 1.76-29.4). Nonneoplastic/non-idiopathic etiology and hemodynamic instability were associated with the composite outcome of in-hospital mortality, need for emergency cardiac surgery, or pericardiocentesis-related complications (OR 5.75, 95\% CI 1.65-20.01, and OR 5.81, 95\% CI 2.11-15.97, respectively). Multivariate Cox regression analysis adjusted for possible confounding variables (age, coronary artery disease, and hemodynamic instability) showed that neoplastic etiology was independently associated with medium-term mortality (hazard ratio [HR] 4.05, 95\% CI 1.45-11.36). In a real-world population treated with pericardiocentesis for pericardial effusion, in-hospital adverse outcomes and medium-term mortality are consistent, in particular for patients presenting with hemodynamic instability or neoplastic pericardial effusion.
\end{abstract}

Keywords Pericardiocentesis $\cdot$ Pericardial effusion $\cdot$ Cardiac tamponade $\cdot$ Mortality $\cdot$ Neoplastic effusion

Giuseppe Boriani

giuseppe.boriani@unimore.it

1 Cardiology Division, Department of Biomedical, Metabolic and Neural Sciences, University of Modena and Reggio Emilia, Policlinico di Modena, Via del Pozzo, 71, 41124 Modena, Italy

2 Oncology Division, University of Modena and Reggio Emilia, Policlinico di Modena, Modena, Italy

3 Clinical and Experimental Medicine PhD Program, University of Modena and Reggio Emilia, Modena, Italy

\section{Introduction}

Pericardial effusion is a clinical condition characterized by an abnormal accumulation of fluid in the pericardial space. Given the limited space in the pericardial cavity, fluid accumulation can severely impact patients' hemodynamic status with dramatic clinical consequences. Multiple different etiologies can be recognized including iatrogenic, infective, neoplastic, autoimmune, idiopathic, and several others less represented (e.g., congestive heart failure, uremic, liver cirrhosis, aortic syndromes) [1-10]. Regardless of the etiology, pericardial effusion is usually treated by percutaneous pericardiocentesis, which has a twofold purpose, therapeutic and diagnostic [11]. The procedure can be performed both in the urgency/emergency clinical setting (i.e., cardiac tamponade) 
or it can be programmed when the instrumental findings (echocardiography, cardiac tomography [CT] scan or magnetic resonance imaging [MRI]) and clinical characteristics of the patient suggest the possibility of an evolution towards cardiac tamponade $[12,13]$. In clinical practice, chemical-physical examination, cytology and microbiology tests are essential steps in the diagnostic process aimed at delineating the exact etiology of pericardial effusion [14-17]. It has been reported that neoplastic etiology is associated with higher mortality compared to the others [18]. However, given the great heterogeneity of patients included in the studies, it is not easy to identify potential predictors of mortality, and data available in the literature are scanty.

The aim of our study is to investigate the clinical characteristics of patients undergoing percutaneous pericardiocentesis and to evaluate possible predictors of adverse clinical outcomes and medium-term mortality according to different etiologies of pericardial effusion.

\section{Methods}

We performed an observational, retrospective, single-center study. All patients undergoing percutaneous pericardiocentesis between January 2010 and December 2019 at the University Hospital of Modena were included in the study. The study protocol (No. 0007514/20) was approved by the local IRB/Ethics Committee in compliance with national regulations.

\section{Patient and pericardial effusion analysis}

Baseline and follow-up data were obtained from electronic and paper medical records and were stored in a customized database. Patients' data were obtained through the review of electronic medical records. We retrospectively collected the following variables: age, sex, full clinical and medication history, etiological diagnosis of the pericardial effusion, clinical presentation features (cardiac tamponade, urgency/ emergency, hemodynamic instability, and atrial fibrillation), date of the pericardiocentesis, need for acute intra-hospital surgery, pericardiocentesis-related complications, puncture site, date of death and date of last clinical contact. All the patients underwent percutaneous pericardiocentesis, guided by echocardiography and/or fluoroscopy and had extended pericardial drainage until there was less than $50 \mathrm{ml}$ of fluid remaining.

For the purpose of this analysis, the study population was divided into three groups according to different etiologies of pericardial effusion: (1) neoplastic, (2) idiopathic and (3) all other etiologies. The etiology of pericardial effusion was classified as follows: (i) neoplastic effusion was diagnosed if pericardial fluid cytology included atypical/malignant cells or negative cytological analysis with instrumental (CT scan and/or MRI) evidence of pericardial involvement with known or newly diagnosed neoplasia; (ii) iatrogenic effusion if patients have undergone invasive medical tests or maneuvers that have caused pericardial effusion (e.g., coronary intervention, pacemaker implantation, valvuloplasty), or patients with proven overdosed anticoagulation therapy, causing hemopericardium; (iii) acute myocardial infarction (AMI) mechanical complications included patients with diagnosis of acute-subacute myocardial infarction and mechanical complications such as myocardial wall rupture, (iv) pericardial effusion secondary to acute aortic syndrome included patients with diagnosis of aortic dissection, proven by transthoracic or transesophageal echocardiogram or CT angiography, causing hemopericardium, (v) infective effusion included patients who had positive pericardial fluid culture for bacterial, mycobacterial or positive serological tests for viral etiology, (vi) autoimmune disease was considered in patients with signs of inflammation in a clinical setting of polyserositis, in known or newly diagnosed autoimmune disease, (vii) uremic pericardial effusion was diagnosed when blood urea nitrogen was $>60 \mathrm{mg} / \mathrm{dl}$ or the patient was dialysis dependent in the absence of other identifiable causes, (viii) congestive heart failure group included patients with pericardial effusion in the presence of clinical symptoms and reduced left ventricular ejection fraction $(\mathrm{LVEF})<50 \%$, if any other causes were excluded, ix) liver cirrhosis was considered in those patients diagnosed with decompensated liver cirrhosis, transudative pericardial fluid and after the exclusion of other possible causes, and (x) idiopathic etiology was diagnosed when all the other possible diagnosis were excluded. Hemodynamic instability was defined as: cardiac arrest, systolic blood pressure (BP) $<90 \mathrm{mmHg}$ or vasopressors required to achieve a $\mathrm{BP} \geq 90 \mathrm{mmHg}$ despite adequate filling status and end-organ hypoperfusion or systolic $\mathrm{BP}<90 \mathrm{mmHg}$ or systolic $\mathrm{BP}$ drop $\geq 40 \mathrm{mmHg}$, lasting longer than $15 \mathrm{~min}$ and not caused by new-onset arrhythmia, hypovolemia, or sepsis [19]. Cardiac tamponade was diagnosed in the presence of typical clinical and echocardiographic signs or CT/MRI imaging. An estimated glomerular filtration rate $(\mathrm{eGRF})<60 \mathrm{ml} / \mathrm{min} / 1.73 \mathrm{~m}^{2}$ identified patients with chronic kidney disease (CKD) according to the "Kidney Disease: Improving Global Outcome (KDIGO)" classification [20]. Acute surgery included patients who underwent a surgical intervention after percutaneous pericardiocentesis, as definitive treatment (e.g., myocardial wall rupture surgical repair or pericardial window).

\section{Study outcomes}

The primary outcome of the study was all-cause mortality. Other outcomes of interest were in-hospital mortality and the composite outcome of in-hospital mortality, 
need for emergency cardiac surgery or pericardiocentesisrelated complications.

\section{Statistical analysis}

Continuous variables are expressed as mean \pm standard deviation or median with interquartile range (IQR) for skewed distributions. Among-group comparisons were made using a non-parametric test (Kruskal-Wallis test). Categorical variables are expressed as counts and percentages. Between-group comparisons were made using a Chi-square test or a Fisher's exact test if any expected cell count was less than five. Prognostic factors for in-hospital mortality and for the composite outcome were assessed by logistic univariate regression analysis. Medium-term survival curves were built using the Kaplan-Meier method. Comparisons were made using the log-rank test. A multivariate Cox proportional hazards regression analysis adjusted for age, hemodynamic instability and presence of coronary artery disease was performed to evaluate the effect of different pericardial effusion's etiologies on allcause death during follow-up. In the comparison between etiologies, the idiopathic category (group 1) was used as the reference. In all analyses, a $P$ value $<0.05$ was considered statistically significant. Statistical analysis was performed using SPSS $^{\circledR}$ (version 26).

\section{Results}

Eighty-one consecutive patients who underwent pericardiocentesis between January 2010 and December 2019 were included in this analysis. Patient clinical characteristics stratified by three different etiological groups are shown in Table 1. Overall, median age was 71.4 years (IQR $58.1-78.1$ years) and 51/81 (63\%) patients were male. Neoplastic etiology group was younger $(p=0.05)$ and tended to be characterized by a lower prevalence of comorbidities, in particular the absence of concomitant coronary artery disease $(p=0.004)$. No differences were found in antithrombotic treatment among different groups. Most of the procedures were performed in an urgency/emergency setting $(76.5 \%)$ and the main indication was cardiac tamponade (77.8\%). Hemodynamic instability at admission was significantly lower in the idiopathic group $(p<0.001)$. Different etiologies of pericardial effusion are specified in Table 2. The most common etiology was idiopathic (33.3\%) followed by neoplastic (22.2\%) and iatrogenic (18.5\%). Lung cancer was the most represented etiology in the neoplastic subgroup (50\%). Among the iatrogenic group, 8 (53.3\%) patients had coronary perforation during percutaneous coronary interventions and $2(13.3 \%)$ patients experienced anticoagulant overdose.

Major adverse events are shown in Table 3. During a mean follow-up of $17.1 \pm 26.5$ months, 36 (44.4\%) patients died. The neoplastic group showed a significantly higher mortality rate compared to other groups $(\mathrm{p}<0.001)$.

Table 1 Baseline patients' characteristics stratified by different etiologies

\begin{tabular}{|c|c|c|c|c|c|}
\hline & Total $(N=81)$ & Neoplastic $(N=18)$ & Idiopathic $(N=27)$ & Others $(N=36)$ & $p$ value \\
\hline Age, median [IQR] & $71.4[58.1-78.1]$ & $66.7[56.8-75.4]$ & $66.8[53.1-76.5]$ & $76.1[61.8-81.5]$ & 0.05 \\
\hline Female, $n(\%)$ & $30(37.0)$ & $7(38.9)$ & $8(29.6)$ & $15(41.7)$ & 0.64 \\
\hline \multicolumn{6}{|l|}{ Comorbidities, $n(\%)$} \\
\hline Arterial hypertension & $48(60.8)$ & $8(47.1)$ & $17(63.0)$ & $23(65.7)$ & 0.42 \\
\hline Dyslipidemia & $28(35.4)$ & $4(23.5)$ & $11(40.7)$ & $13(37.1)$ & 0.49 \\
\hline Diabetes mellitus & $17(21.5)$ & $1(5.9)$ & $5(18.5)$ & $11(31.4)$ & 0.11 \\
\hline Coronary artery disease & $17(21.5)$ & $0(0.0)$ & $4(14.8)$ & $13(37.1)$ & 0.004 \\
\hline Atrial fibrillation & $23(28.4)$ & $3(16.7)$ & $7(25.9)$ & $13(36.1)$ & 0.31 \\
\hline CKD & $24(30.8)$ & $2(12.5)$ & $8(29.6)$ & $14(40.0)$ & 0.13 \\
\hline Antithrombotic treatment, $n(\%)$ & $25(30.9)$ & $4(22.2)$ & $7(25.9)$ & $14(38 . .8)$ & 0.25 \\
\hline VKA & 10 & 0 & 2 & 8 & \\
\hline NOAC & 6 & 0 & 3 & 3 & \\
\hline LMWH or calcium heparin & 9 & 4 & 2 & 3 & \\
\hline Hemodynamic instability, $n(\%)$ & $29(36.3)$ & $4(22.2)$ & $4(14.8)$ & $21(60.0)$ & $<0.001$ \\
\hline Urgency, $n(\%)$ & $62(76.5)$ & $12(66.7)$ & $18(66.7)$ & $32(88.9)$ & 0.06 \\
\hline Cardiac tamponade, $n(\%)$ & $63(77.8)$ & $12(66.7)$ & $20(74.1)$ & $31(86.1)$ & 0.23 \\
\hline
\end{tabular}

$C K D$ chronic kidney disease, $I Q R$ interquartile range, $L M W H$ low-molecular-weight heparin, NOAC non-vitamin $\mathrm{K}$ oral anticoagulants, $V K A$ vitamin $\mathrm{K}$ antagonist 
Table 2 Etiologies of pericardial effusion at admission

\begin{tabular}{|c|c|}
\hline Etiologies, $n(\%)$ & Total, $N=81$ \\
\hline Idiopathic & $27(33.3)$ \\
\hline Neoplastic & $18(22.2)$ \\
\hline Lung cancer & 9 \\
\hline Gastric cancer & 2 \\
\hline Hematological malignancies & 2 \\
\hline Breast cancer & 1 \\
\hline Larynx & 1 \\
\hline Colon & 1 \\
\hline Ovary & 1 \\
\hline Mesothelioma & 1 \\
\hline Iatrogenic & $15(18.5)$ \\
\hline Coronary angioplasty & 8 \\
\hline Coronary angiography & 1 \\
\hline Valvuloplasty & 1 \\
\hline Radiofrequency ablation & 1 \\
\hline Temporary PM removal & 1 \\
\hline Anticoagulant therapy overdose & 2 \\
\hline Percutaneous interatrial defect closure & 1 \\
\hline AMI complications & $6(7.4)$ \\
\hline Infective & $4(4.9)$ \\
\hline Acute heart failure & $4(4.9)$ \\
\hline Autoimmune & $3(3.7)$ \\
\hline Uremia & $1(1.2)$ \\
\hline Liver cirrhosis & $1(1.2)$ \\
\hline Aortic dissection & $1(1.2)$ \\
\hline Others & $1(1.2)$ \\
\hline
\end{tabular}

$A M I$ acute myocardial infarction, $P M$ pacemaker

In-hospital mortality was $14.8 \%$ without any significant difference among groups. There were $5(6.2 \%)$ major pericardiocentesis-related complications: 1 (1.25\%) pleural puncture, 2 (2.5\%) liver puncture with hemoperitoneum, $1(1.25 \%)$ right ventricle puncture with self-resolution and without effect on the quantity of pericardial fluid and 1 $(1.25 \%)$ infective complication treated with targeted antibiotic therapy. Fourteen out of 81 (17.3\%) patients required emergency cardiac surgery. As shown in the Kaplan-Meier survival curve (Fig. 1), patients with malignant pericardial effusion's etiology had a significantly worse prognosis at follow-up (log-rank test $p=0.001$ ). At univariate logistic regression analysis (Table 4), only hemodynamic instability was associated with in-hospital mortality (OR 7.20; CI $1.76-29.40 ; p=0.006$ ). The etiology of pericardial effusion instead was not. With regard to the in-hospital composite outcome, hemodynamic instability (OR 5.81, 95\% CI 2.11-15.97, $p=0.001$ ) and non-idiopathic/non-neoplastic group (OR 5.75, 95\% CI 1.65-20.01, $p=0.006$ ) were associated with worse outcome (Table 4). At multivariate Cox proportional hazards regression analysis adjusted for age, hemodynamic instability and the presence of coronary artery disease, neoplastic etiology was independently associated with a higher risk of all-cause death during follow-up (HR $4.05,95 \%$ CI 1.45-11.36, $p=0.008$ ) (Table 5).

\section{Discussion}

Percutaneous pericardiocentesis is the procedure of choice for the removal of severe pericardial effusion presenting with or without cardiac tamponade. This procedure plays an important role for both diagnostic and therapeutic purposes $[21,22]$. The present study analyzes a real-world population undergoing pericardiocentesis and aims at improving patients' clinical management, according to their specific pericardial effusion's etiology, and defining predictors of worse clinical outcomes. The major findings of this analysis are the following: first, percutaneous pericardiocentesis is fairly safe showing complications in a limited number of patients; second, hemodynamic instability is a predictor of in-hospital mortality; third, patients presenting with pericardial effusion unrelated to neoplastic etiology were associated with a higher rate of in-hospital mortality, need for emergency cardiac surgery or pericardiocentesis-related complications; fourth, neoplastic etiology was independently associated with a higher risk of all-cause death during the follow-up. Nowadays, percutaneous pericardiocentesis is a safe procedure albeit it is not easy to perform. The most feared complications are ventricle, liver or pulmonary puncture. To reduce the rate of these unwished events, the use of echo- or fluoroscopy-guided technique to better perform the procedure is recommended. In our study, peri-procedural complications occurred in a limited number of patients (6.2\%). This result is in line with European data, (4-10\%) and with the largest studies (5.9\%) [3]. The diagnostic phase
Table 3 Major adverse events stratified by different etiologies

\begin{tabular}{llllll}
\hline & Total $(N=81)$ & Neoplastic $(N=18)$ & Idiopathic $(N=27)$ & Others $(N=36)$ & $p$ value \\
\hline All-cause death & $36(44.4)$ & $14(77.8)$ & $6(22.2)$ & $16(44.4)$ & 0.001 \\
In-hospital mortality & $12(14.8)$ & $3(16.7)$ & $2(7.4)$ & $7(19.4)$ & 0.40 \\
Major complications & $5(6.2)$ & $0(0.0)$ & $1(3.7)$ & $4(11.1)$ & 0.29 \\
$\begin{array}{l}\text { Need of emergency } \\
\text { cardiac surgery }\end{array}$ & $14(17.3)$ & $3(16.7)$ & $2(7.4)$ & $9(25.0)$ & 0.19 \\
\hline
\end{tabular}




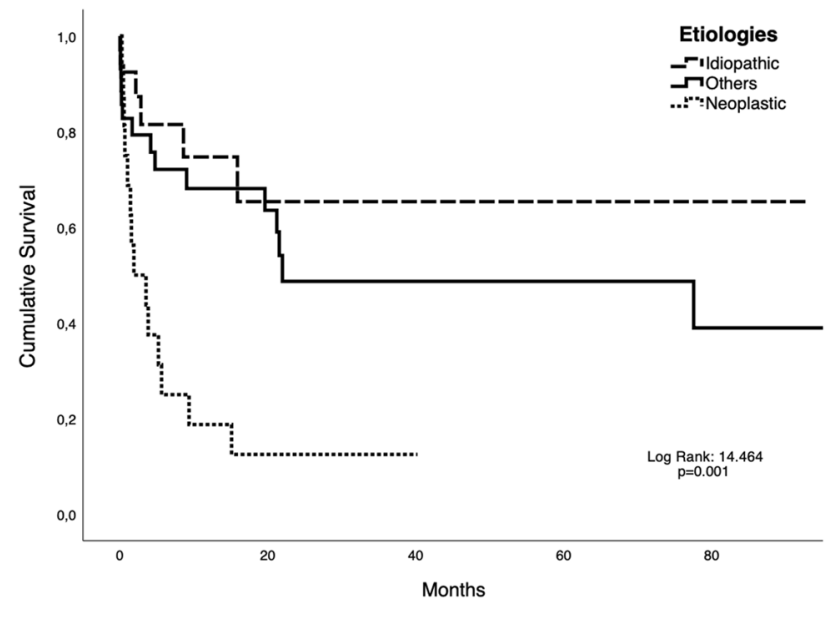

Fig. 1 Kaplan Meier curves for freedom from all-cause mortality

is certainly the most challenging when managing patients with pericardial effusion. This is due to incomplete or insufficient diagnostic tests that do not always allow for the discovery of the real etiology of the effusion. Cases with idiopathic etiology are prevalent in our study, followed by those of neoplastic origin: this may be explained also by the fact that our institution includes the Provincial Oncological Reference Center. Other studies in the literature have shown a predominance of the neoplastic etiology [7]. However, it has to be underlined that may exist a perception about underestimating neoplastic effusions, suspecting that the cytology of the pericardial fluid is sometimes insufficient to confirm the diagnosis. For this reason, the question arises as to whether pericardial biopsy must be carried out in case of strong suspicion [7]. This scenario is in line with our data but the lesser number of patients observed may have aggravated this aspect.

Regarding outcomes, we predictably have observed that hemodynamic instability increases the risk of intra-hospital mortality rate regardless of the etiology of the pericardial effusion; the higher intra-hospital mortality rate is probably a consequence of the fact that patients with acute heart conditions often come to hospital and therefore must undergo an emergency life-saving procedure, which inevitably increases the patient's clinical risk.

The in-hospital mortality rate that emerged in our case series is, however, comparable with that of other studies published in the literature (14-19\%) [3, 7, 8, 23-25]. Orbach et al. [8] found that in-hospital mortality rate increases in the group of patients with pericardial effusion secondary to AMI mechanical complications and coagulopathies/ongoing anticoagulant treatment; this evidence was not observed in our study due to the scarce amount of events of this type (respectively, 6 cases of AMI complications and 2 cases of hemopericardium caused by anticoagulant treatment). In particular, pericardial effusion secondary to anticoagulant therapy is a clinical condition that has to be considered nowadays, with the extensive use of NOACs in the older population, mainly prescribed for atrial fibrillation [26, 27]. The group of patients presenting with pericardial effusion unrelated
Table 4 Logistic regression analysis for predictors of in-hospital mortality and in-hospital composite outcome (in-hospital mortality, need of emergency cardiac surgery or pericardiocentesis-related complications)

\begin{tabular}{|c|c|c|c|c|c|c|}
\hline & \multicolumn{3}{|c|}{ In-hospital mortality* } & \multicolumn{3}{|c|}{ Composite outcome* } \\
\hline & OR & $95 \% \mathrm{CI}$ & $P$ value & OR & $95 \% \mathrm{CI}$ & $P$ value \\
\hline \multicolumn{7}{|l|}{ Etiology } \\
\hline Idiopathic (reference) & - & - & - & - & - & - \\
\hline Others & 3.01 & $0.57-15.86$ & 0.19 & 5.75 & $1.65-20.01$ & 0.006 \\
\hline Neoplastic & 2.50 & $0.37-16.72$ & 0.34 & 2.21 & $0.50-9.72$ & 0.29 \\
\hline Age & 1.05 & $0.99-1.12$ & 0.07 & 0.99 & $0.96-1.03$ & 0.97 \\
\hline Female sex & 0.83 & $0.23-3.02$ & 0.77 & 0.79 & $0.29-2.07$ & 0.63 \\
\hline Hypertension & 2.15 & $0.53-8.68$ & 0.28 & 0.91 & $0.35-2.35$ & 0.84 \\
\hline Dyslipidemia & 1.36 & $0.39-4.79$ & 0.62 & 0.67 & $0.25-1.83$ & 0.44 \\
\hline Diabetes mellitus & 1.26 & $0.30-5.29$ & 0.75 & 1.47 & $0.49-4.43$ & 0.49 \\
\hline Coronary artery disease & 2.07 & $0.54-7.97$ & 0.29 & 2.75 & $0.92-8.25$ & 0.07 \\
\hline Atrial fibrillation & 2.02 & $0.57-7.19$ & 0.28 & 1.09 & $0.39-3.03$ & 0.86 \\
\hline Chronic kidney disease & 1.34 & $0.35-5.10$ & 0.66 & 1.00 & $0.36-2.77$ & 1.00 \\
\hline Antithrombotic treatment & 1.63 & $0.47-5.74$ & 0.45 & 0.48 & $0.16-1.40$ & 0.18 \\
\hline Hemodynamic instability & 7.20 & $1.76-29.40$ & 0.006 & 5.81 & $2.11-15.97$ & 0.001 \\
\hline Urgency & 3.88 & $0.47-32.23$ & 0.21 & 3.37 & $0.88-12.79$ & 0.07 \\
\hline Cardiac tamponade & 3.59 & $0.43-29.93$ & 0.24 & 3.07 & $0.81-11.75$ & 0.10 \\
\hline Subxiphoid puncture site & 0.87 & $0.23-3.29$ & 0.85 & 0.77 & $0.25-2.35$ & 0.65 \\
\hline
\end{tabular}

* Analysis presented is unadjusted univariate logistic regression analysis; OR=odds ratio; CI confidence interval 
Table 5 Multivariate Cox regression analysis for all-cause death according to different etiologies

\begin{tabular}{lccc}
\hline & \multicolumn{3}{l}{ Adjusted analysis* } \\
\cline { 2 - 4 } & HR & $95 \%$ CI & $P$ value \\
\hline Etiology & & & \\
Idiopathic (reference) & - & - & - \\
Others & 0.79 & $0.23-2.61$ & 0.70 \\
Neoplastic & 4.05 & $1.45-11.36$ & 0.008 \\
\hline
\end{tabular}

*Adjusted analysis for age, hemodynamic instability and the presence of coronary artery disease at baseline; $H R$ hazard ratio, $C I$ confidence interval

to neoplastic etiology was composed of AMI mechanical complications and iatrogenic etiology: these patients are often burdened by older age, important comorbidities and poorer clinical status. Probably, these can be the reasons for the higher rate of in-hospital mortality, need for emergency cardiac surgery or pericardiocentesis-related complications that we observed in this subgroup of patients.

Patients with neoplastic effusion instead have a higher mortality rate during the follow-up. Often the pericardial effusion develops gradually in the context of advanced cancer, therefore, patients show less frequently signs and symptoms of acute cardiac tamponade, but their short/ medium-term prognosis is poorer because of the neoplastic pathology itself [28]. Due to the poor prognosis of patients with advanced malignancy and associated pericardial effusion, the primary therapeutic goal in these patients should be the improvement of symptoms and quality of life. In this complex setting, a multidisciplinary team approach, including cardiologists, oncologists, cardio-thoracic surgeons and palliative care physicians, is advisable and should be encouraged [29].

The current analysis did not cover the period of COVID19 pandemic, and therefore, did not take into account that pericardial effusion is one of the manifestations of COVID19 , with the possibility of cardiac tamponade in the setting of a severe clinical course [30]. Future studies will have to consider also this etiology of pericardial effusions [30-33].

\section{Limitations of the study}

The present study has specific limitations that need to be addressed. First, our cohort represents a single-medical center experience with a small sample size, thus limiting the generalization of the results. As any retrospective study, the statistical power of the analysis is limited due to the absence of a prospective design and follow-up. The small number of patients did not allow us to investigate any possible difference in outcome among specific subtypes of malignancy.
Moreover, prognostic factors for in-hospital mortality and for the composite outcome were assessed only by logistic univariate regression analysis limiting the power of the association. Cox regression model was adjusted only for age, hemodynamic instability and the presence of coronary artery disease to avoid possible overfitting with the small number of events.

Lastly, the relatively short follow-up period could have limited power to associate the different etiology groups with outcomes.

\section{Conclusions}

The most common etiology of pericardial effusion requiring percutaneous pericardiocentesis in our contemporary realworld population is idiopathic, and the most common clinical presentation is cardiac tamponade. In-hospital adverse outcomes and medium-term mortality are not negligible. Hemodynamic instability was associated with in-hospital mortality and non-neoplastic/non-idiopathic etiology and hemodynamic instability were associated with the composite outcome of in-hospital mortality, need for emergency cardiac surgery or pericardiocentesis-related complications. Finally, neoplastic etiology was associated with higher medium-term mortality.

Funding No funding was received for this work.

\section{Compliance with ethical standards}

Conflict of interest GB received small speaker's fees from Medtronic, Boston, Biotronik, Boehringer, and Bayer, outside of the submitted work. The other authors declare no conflict of interest.

Statement of human and animal rights All the procedures performed in the patients of this study were in accordance with the ethical standards of the Institutional Ethic Committee for Clinical Research and with the 1964 Declaration of Helsinki and its later amendments or comparable ethical standards.

Informed consent All the patients released informed consent for the procedures evaluated in this study.

\section{References}

1. Ma W, Liu J, Zeng Y et al (2012) Causes of moderate to large pericardial effusion requiring pericardiocentesis in $140 \mathrm{Han}$ Chinese patients. Herz 37:183-187. https://doi.org/10.1007/s0005 9-011-3428-5

2. Sagristà-Sauleda J, Mercé J, Permanyer-Miralda G et al (2000) Clinical clues to the causes of large pericardial effusions. Am J Med 109:95-101. https://doi.org/10.1016/s0002-9343(00)00459 $-9$ 
3. Strobbe A, Adriaenssens T, Bennett J et al (2017) Etiology and long-term outcome of patients undergoing pericardiocentesis. J Am Heart Assoc. https://doi.org/10.1161/JAHA.117.007598

4. Levy PY, Corey R, Berger P et al (2003) Etiologic diagnosis of 204 pericardial effusions. Medicine (Baltimore) 82:385-391. https ://doi.org/10.1097/01.md.0000101574.54295.73

5. Corey GR, Campbell PT, Van Trigt P et al (1993) Etiology of large pericardial effusions. Am J Med 95:209-213. https://doi. org/10.1016/0002-9343(93)90262-n

6. Gad MM, Elgendy IY, Mahmoud AN et al (2020) Temporal trends, outcomes, and predictors of mortality after pericardiocentesis in the United States. Catheter Cardiovasc Interv 95:375-386. https://doi.org/10.1002/ccd.28588

7. Sánchez-Enrique C, Nuñez-Gil IJ, Viana-Tejedor A et al (2016) Cause and long-term outcome of cardiac tamponade. Am J Cardiol 117:664-669. https://doi.org/10.1016/j.amjcard.2015.11.023

8. Orbach A, Schliamser JE, Flugelman MY et al (2016) Contemporary evaluation of the causes of cardiac tamponade: Acute and long-term outcomes. Cardiol J 23:57-63. https://doi.org/10.5603/ CJ.a2015.0041

9. Sethi A, Singbal Y, Kodumuri V et al (2018) Inpatient mortality and its predictors after pericardiocentesis: an analysis from the Nationwide Inpatient Sample 2009-2013. J Interv Cardiol 31:815-825. https://doi.org/10.1111/joic. 12563

10. Morello F, Santoro M, Fargion AT et al (2020) Diagnosis and management of acute aortic syndromes in the emergency department. Intern Emerg Med. https://doi.org/10.1007/s11739-02002354-8

11. Maggiolini S, De Carlini CC, Imazio M (2018) Evolution of the pericardiocentesis technique. J Cardiovasc Med (Hagerstown) 19:267-273. https://doi.org/10.2459/JCM.0000000000000649

12. Patel N, Rafique AM, Eshaghian S et al (2013) Retrospective comparison of outcomes, diagnostic value, and complications of percutaneous prolonged drainage versus surgical pericardiotomy of pericardial effusion associated with malignancy. Am J Cardiol 112:1235-1239. https://doi.org/10.1016/j.amjcard.2013.05.066

13. Imazio M, Gaido L, Battaglia A et al (2017) Contemporary management of pericardial effusion: practical aspects for clinical practice. Postgrad Med 129:178-186. https://doi.org/10.1080/00325 481.2017.1285676

14. Ristić AD, Imazio M, Adler Y et al (2014) Triage strategy for urgent management of cardiac tamponade: a position statement of the european society of cardiology working group on myocardial and pericardial diseases. Eur Heart J 35:2279-2284. https://doi. org/10.1093/eurheartj/ehu217

15. Karatolios K, Pankuweit S, Maisch B (2013) Diagnostic value of biochemical biomarkers in malignant and non-malignant pericardial effusion. Heart Fail Rev 18:337-344. https://doi.org/10.1007/ s10741-012-9327-x

16. Meyers DG, Bouska DJ (1989) Diagnostic usefulness of pericardial fluid cytology. Chest 95:1142-1143. https://doi.org/10.1378/ chest.95.5.1142

17. Adler Y, Charron P, Imazio M et al (2015) 2015 ESC Guidelines for the diagnosis and management of pericardial diseases: The task force for the diagnosis and management of pericardial diseases of the european society of cardiology (ESC) endorsed by: the european association for cardio-thoracic surgery (EACTS). Eur Heart J 36:2921-2964. https://doi.org/10.1093/eurheartj/ ehv318

18. Santas E, Núñez J (2016) Prognostic implications of pericardial effusion: The importance of underlying etiology. Int J Cardiol 202:407. https://doi.org/10.1016/j.ijcard.2015.09.051

19. Konstantinides SV, Meyer G (2019) The 2019 ESC guidelines on the diagnosis and management of acute pulmonary embolism. Eur Heart J 40:3453-3455. https://doi.org/10.1093/eurheartj/ehz726
20. Stevens PE, Levin A, Members KDIGOCKDGDWG (2013) Evaluation and management of chronic kidney disease: synopsis of the kidney disease: improving global outcomes 2012 clinical practice guideline. Ann Intern Med 158:825-830. https://doi. org/10.7326/0003-4819-158-11-201306040-00007

21. Imazio M, De Ferrari GM (2020) Cardiac tamponade: an educational review. Eur Heart J Acute Cardiovasc Care. https://doi. org/10.1177/2048872620939341

22. Lazaros G, Antonopoulos AS, Imazio M et al (2019) Clinical significance of pleural effusions and association with outcome in patients hospitalized with a first episode of acute pericarditis. Intern Emerg Med 14:745-751. https://doi.org/10.1007/s1173 9-019-02041-3

23. El Haddad D, Iliescu C, Yusuf SW et al (2015) Outcomes of cancer patients undergoing percutaneous pericardiocentesis for pericardial effusion. J Am Coll Cardiol 66:1119-1128. https:// doi.org/10.1016/j.jacc.2015.06.1332

24. Albugami S, Al-Husayni F, AlMalki A et al (2020) Etiology of pericardial effusion and outcomes post pericardiocentesis in the western region of saudi arabia: a single-center experience. Cureus 12:e6627. https://doi.org/10.7759/cureus.6627

25. Cheong XP, Law LKP, Seow SC et al (2019) Causes and prognosis of symptomatic pericardial effusions treated by pericardiocentesis in an Asian academic medical centre. Singapore Med J. https:// doi.org/10.11622/smedj.2019065

26. Malavasi VL, Fantecchi E, Gianolio L et al (2019) Atrial fibrillation in patients with active malignancy and use of anticoagulants: Under-prescription but no adverse impact on all-cause mortality. Eur J Intern Med 59:27-33. https://doi.org/10.1016/j. ejim.2018.10.012

27. Sbrana F, Mannucci F, Airò E et al (2018) Cardiac tamponade due to apixaban therapy in patient with unknown pericardial hemangioma. Intern Emerg Med 13:297-299. https://doi.org/10.1007/ s11739-017-1736-3

28. De Filippo O, Gatti P, Rettegno S et al (2019) Is pericardial effusion a negative prognostic marker? Meta-analysis of outcomes of pericardial effusion. J Cardiovasc Med (Hagerstown) 20:39-45. https://doi.org/10.2459/JCM.0000000000000720

29. Imazio M, Adler Y (2013) Management of pericardial effusion. Eur Heart J 34:1186-1197. https://doi.org/10.1093/eurheartj/ ehs372

30. Farina A, Uccello G, Spreafico M et al (2020) SARS-CoV-2 detection in the pericardial fluid of a patient with cardiac tamponade. Eur J Intern Med 76:100-101. https://doi.org/10.1016/j. ejim.2020.04.045

31. Imazio M, Brucato A, Lazaros G et al (2020) Anti-inflammatory therapies for pericardial diseases in the COVID-19 pandemic: safety and potentiality. J Cardiovasc Med (Hagerstown) 21:625629. https://doi.org/10.2459/JCM.0000000000001059

32. Hua A, O'Gallagher K, Sado D et al (2020) Life-threatening cardiac tamponade complicating myo-pericarditis in COVID-19. Eur Heart J 41:2130. https://doi.org/10.1093/eurheartj/ehaa253

33. Di Domenico SL, Coen D, Bergamaschi M et al (2020) Clinical characteristics and respiratory support of 310 COVID-19 patients, diagnosed at the emergency room: a single-center retrospective study. Intern Emerg Med. https://doi.org/10.1007/s11739-02002548-0

Publisher's Note Springer Nature remains neutral with regard to jurisdictional claims in published maps and institutional affiliations. 